\title{
Isotopic communication across the water - rock interface: Preservation in solids and signatures in fluids
}

\author{
Jennifer L. Druhan ${ }^{1, *}$, and Nicole Fernandez ${ }^{1,2}$ \\ ${ }^{1}$ Department of Geology, University of Illinois Urbana Champaign, 1301 W. Green St., Urbana, IL, \\ 61802, United States \\ ${ }^{2}$ Institut de Physique du Globe de Paris, Paris, 75321, France
}

\begin{abstract}
Water - rock interactions are characteristically associated with isotopic fractionation between phases, yet their remains significant uncertainty in the degree to which these isotope ratios record the conditions of mineral formation or equilibration, and thus their fidelity as proxy records. Here, we present a novel generic modeling framework allowing sub-grid scale tracking of the isotopic signatures recorded in newly formed minerals over the timescale of precipitation and continued isotopic exchange between phases. We demonstrate that the surface area of the solid phase strongly influences the extent to which isotopes reflect the conditions of formation through time.
\end{abstract}

\section{Introduction}

Weathering reactions occur in systems that are out of equilibrium. Commonly, these transformations are limited by some time-dependent maximum rate of mass transfer that keeps them from reestablishing equilibrium instantaneously [1]. For the stable isotope ratios that are partitioned between fluid and solid phases as a result of such water-rock interaction, the characteristic fractionation factors creating these signatures are either associated with differences in the rate constants (i.e. kinetic fractionation) or differences in equilibrium distributions (i.e. equilibrium fractionation). Thus, a broad range of fields have grappled (both directly and implicitly) with the extent to which isotopic signatures of minerals reflect the thermodynamics of the environment in which they formed, or rate of formation, and how these signatures change through time [2].

At one extreme, many fields utilize the isotopic composition of solids to infer the equilibrium conditions of the environment at the time of formation. For example, soil carbonate $\delta^{13} \mathrm{C}$ is used to infer atmospheric $\mathrm{pCO}_{2}$ at the time the mineral was precipitated [3]. Similar principles are used to infer temperature proxies, such as for trace element ratios and $\delta^{18} \mathrm{O}$ of marine carbonates, cave speleothems and corals. At the other extreme, many disciplines necessarily assume that the isotope ratio of a mineral formed from solution reflects kinetic controls on the rate of formation. An example in which this

\footnotetext{
* Corresponding author: jdruhan@illinois.edu
} 
behavior is clearly shown is in the reductive precipitation of redox sensitive species, such as the $\delta^{57} \mathrm{Fe}$ fractionation imparted during iron oxide formation and the $\delta^{34} \mathrm{~S}$ signature of biologically regulated pyrite formation $[4,5]$. These predominantly biological signatures are now being leveraged in novel application to other 'mid-mass' isotope systems such as $\delta^{53} \mathrm{Cr}$ and $\delta^{238} \mathrm{U}$ to track contaminant (re)mobilization [6].

Given that isotopic ratios of minerals can either reflect conditions of equilibrium or kinetically regulated formation, isotopic proxies are often limited by the uncertainty associated with mixed equilibrium and kinetic signatures [7]. Relatedly, there is significant uncertainty associated with the extent to which these signatures at the time of mineral formation are later altered or erased as a result of continued water-rock interaction [8]. To this end, many recent studies have focused on the isotopic composition of newly formed solids and the coevolving fluid phase. In these studies, the intent is to track the preservation of kinetic precipitation signatures and the extent to which this characteristic fractionation is later erased as a function of continued exchange between the fluid and solid surface. A critical aspect of this work concerns the depth into the surface of the solid phase that continues to equilibrate with the surrounding fluid after active mineral precipitation has ceased, and thus the component of the solid that still records the conditions of formation. This isotopic 're-equilibration' or continuing exchange between phases following mineral formation has been demonstrated for magnesium, carbon, strontium and barium [9 - 14]. Several of these studies have attempted to infer the depth into the mineral surface that can isotopically exchange with the fluid phase, thus providing an estimate of the extent to which fluid-rock interaction may alter isotopic records.

We have recently developed and validated a novel reactive transport simulation intended as a generalized and predictive modeling framework for these exchange processes. The model explicitly tracks the component of mass most recently added from the fluid phase to the mineral surface through time, as first described in [15]. Here, we report an update to this approach in which the window of time used for this calculated running average has now been reframed as a user specified input parameter, such that we are capable of constraining the component of mineral volume in communication with the surrounding fluid for a given system. We have developed this model as a means of directly constraining the extent to which fluids impart mixed kinetic and equilibrium signatures and alter those signatures through time in open systems [16]. In the present study, this model is applied to explore a suite of parameter space, thus constraining the subset of environmental conditions under which a generic $\mathrm{AB}$ mineral records equilibrium vs. kinetic signatures, and the timescale over which these signatures are subsequently lost.

\section{Model Framework}

The coupled precipitation of two isotopes of the same element are modeled using a Transition State Theory (TST) rate expression employing a solid solution [15]. This model has been developed and applied previously $[15,17,18]$. Briefly, each isotope is modeled explicitly and subject to its own rate law of the form:

$$
\frac{d\left[{ }^{n} A\right]}{d t}={ }^{n} k S^{n} X\left(\frac{\left[{ }^{n} A\right][B]}{{ }^{n} X^{n} K_{e q}}-1\right)
$$

where the change in activity of the isotope (n) of element $A$ ([A]) over time is the product of the activities of the reactants in solution (here $[\mathrm{A}]+[\mathrm{B}] \rightarrow \mathrm{AB}_{\text {solid }}$ ) relative to the associated (temperature dependent) equilibrium constant $\left(\mathrm{K}_{\mathrm{eq}}\right)$, such that the equilibrium fractionation factor $\alpha_{\text {eq }}$ results from the ratio of the equilibrium constants among isotopes of 
the given element. The overall rate of the reaction as it approaches equilibrium is dictated by the surface area of the mineral $(\mathrm{S})$ and the isotope specific rate constant $(\mathrm{k})$, such that the kinetic fractionation factor $\alpha_{\mathrm{k}}$ results from the ratio of the kinetic rate constants among the isotopes of a given element. In addition, this formulation contains the isotopic mole fractions (X) of the solid phase. As described previously, these isotopic mole fractions represent the solid solution of the two (or more) isotopes of element or molecule A in the solid phase. The key aspect of this formulation for the present study is in the definition of $X$. At one extreme, we may choose to utilize the entire volume of solid phase present in the system, hereafter referred to as the 'bulk' mineral. At the other extreme, we could assume that the value of $\mathrm{X}$ represents the isotopic content of a vanishingly small amount of solid phase instantly formed from the fluid at a given time and immediately removed (i.e. precipitated), such that fractionation proceeds like classic Rayleigh distillation. In between these two extremes, we are compelled to define some fraction of the overall solid phase volume that should be used to calculate isotopic mole fractions for eq. 1. This choice dictates the amount of the solid phase that the fluid can interact with. In an attempt to accomplish this calculation in a logical and self-consistent manner, we develop a feature of the CrunchTope software which enables us to track a running average of the isotopic mole fractions of solid phase most recently precipitated from the fluid through time (Druhan et al., 2013). Thus, the time interval over which we choose to calculate this average dictates the component of the bulk mineral that the fluid is capable of exchanging with $[15-17,19]$.

\section{Parameter Space}

Basic setup: All simulations start at an oversaturation of $\Omega=2$ with a constant ion ratio of $[\mathrm{A}]:[\mathrm{B}]=1.0$ and a rate constant of $\mathrm{k}=10^{-8} \mathrm{~mol} / \mathrm{m}^{2}-\mathrm{s}$. The bulk concentration of $[\mathrm{A}]$ is the sum of two isotopes, $\left[\mathrm{A}^{\prime}\right]$ and $\left[\mathrm{A}^{*}\right]$, where the starting ratio of $\mathrm{A}^{*} / \mathrm{A}^{\prime}=0.02$. This ratio falls within the typical range of many naturally occurring stable isotope distributions, and thus $\mathrm{A}^{*}$ is an appropriate generic representation of a 'minor' isotope. The initial conditions of the system consist of a small volume ( $1 \%$ of REV) of preexisting solid phase $\mathrm{AB}_{\text {solids }}$ with isotopic ratio equal to that of the initial fluid. All simulations incorporate two fractionation factors, an $\alpha_{\mathrm{k}}=0.99$ and an $\alpha_{\mathrm{eq}}=1.00$.

Parameters tested: From this basis we explore a range of parameter space. First, we specify a fixed specific surface area of $1.0 \mathrm{~cm}^{2} / \mathrm{g}$, and vary the time interval over which the isotopic mole fraction (X, eq. 1) is averaged. At one extreme we consider the behavior of a system in which the fluid can 'see' the entire mineral composition, as if there were infinite surface area exposed for isotopic exchange. From this starting point we proceed to regulate this time duration, considering values of 2.4 and 1.2 hours for our running average. Critically, the shorter the time interval over which the running average is calculated, the more the fluid phase is isolated from the interior of the solid and restricted to communication with a thinner surface layer. Following this exercise, we transition to a system with a fixed duration of time over which the running average mineral composition is calculated and instead consider a range of surface areas bounding the original 1.0. Values of 10.0 and $0.1 \mathrm{~cm}^{2} / \mathrm{g}$ are tested. Again, here the key inference is that an increasing surface area for the same time averaging will spread the mass of newly precipitated mineral over a larger space, and thus create a thinner depth into the mineral surface that the isotopes are capable of exchanging with. Thus, from our baseline conditions, both the decreasing time interval and the increasing surface area used in eq. 1 should create greater segregation between the fluid phase and the interior of the solid mineral. 


\section{Results and Discussion}

Our baseline condition illustrates a rapid decrease in solute concentrations through time (Fig. 1A), reaching bulk chemical equilibrium on the order of 6 hours after the start of the simulated precipitation from an oversaturated solution. The corresponding fluid and bulk solid isotope ratios begin at equivalent values (Fig. 1B) which are normalized such that both phases are represented as $0 \%$. As precipitation initiates, the fluid phase is subjected to a kinetic fractionation factor of 0.99 , such that the heavier isotope $A^{*}$ is preferentially left in the residual reactant and thus the isotope ratio of the fluid phase increases. By mass balance, this increase in the residual fluid phase must create a newly formed precipitated mineral that is correspondingly depleted, such that we would observe the mirror image of the fluid phase through time as the isotopic ratio of the new solid. This addition of new solid phase slightly shifts the bulk mineral isotopic value, but the new mass accumulated is small relative to the initial condition and thus the shift is not discernable in the figure.
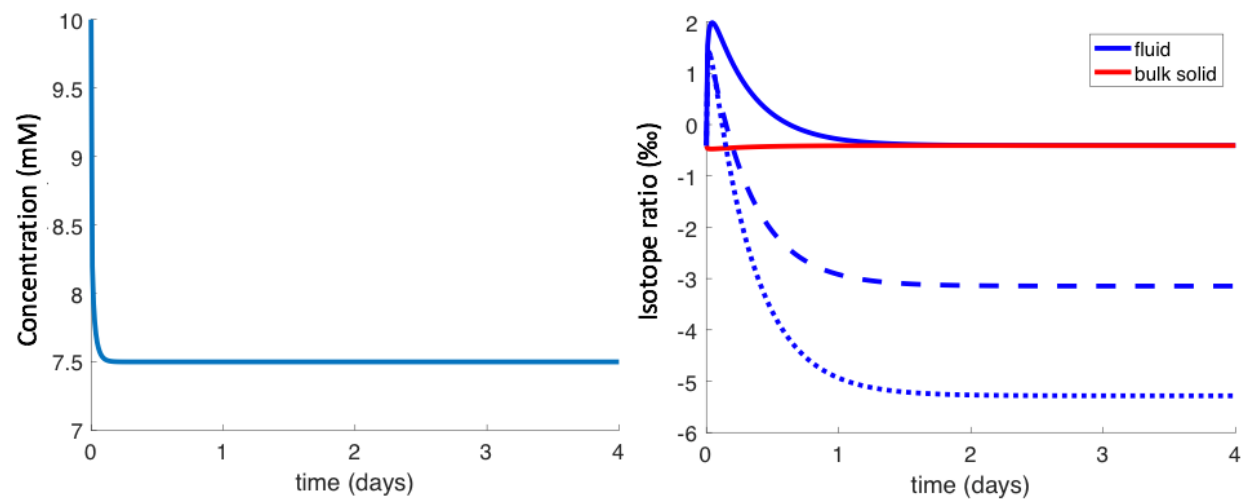

Fig. 1. Timeseries of simulated (A) fluid phase concentration of the initially oversaturated system and (B) the corresponding isotope ratios of the fluid phase (blue) and the bulk solid phase (red). Multiple fluid phase simulations are shown for the isotope ratios which correspond to exchange with the bulk mineral (solid line), exchange with solid phase precipitated over the prior 2.4 hours (dashed line) and 1.2 hours (dotted line). The corresponding influence on the bulk solid isotope ratio as a result of the newly precipitated solid is too small to see at the scale of the figure. The change in fluid concentrations through time are similarly negligible.

The solid lines illustrated in Fig. 1B represent the scenario in which the isotopic composition of the entire mineral is in communication with the surrounding fluid, such that the mole fraction (eq. 1) is set to the bulk mineral value. The result is that the large mass of element $\mathrm{A}$ in the solid phase relative to that in the fluid causes the solid to strongly influence the isotopic composition of the latter. Thus, despite a large enrichment (up to $2 \%$ ) in the fluid phase as a result of kinetic fractionation, the requirement of an equilibrium fractionation factor of 1.00 results in the fluid isotopically exchanging with the bulk solid rapidly after chemical equilibrium is established. In this scenario chemical equilibrium was established approximately 6 hours after the start of the simulation, and subsequent isotopic equilibrium was established in roughly 1.5 days.

Two simulations are also illustrated in which the time window over which the mole fraction (eq. 1) is calculated has been limited to only the mass which has left the fluid phase in the past 2.4 hours, and 1.2 hours. The critical difference in behavior of these simulations relative to the base case scenario is that the fluid is no longer in communication with the bulk solid isotope ratio, and thus after an initial period of kinetic enrichment, the system reestablishes isotopic equilibrium with a small portion of the solid phase which has recently 
been formed. This small portion was initially a negative isotopic ratio mirroring the enrichment in the fluid, and thus the fluid phase establishes an equilibrium fractionation factor of 1.0 with this small, isotopically depleted solid surface. The key result is that if one were to recover a sample of this system and measure both the fluid and solid phase isotope ratios using standard practices (i.e. no sequential dissolution or partitioning of the solid phase) then it would appear that this system is out of equilibrium, when in fact the mineral is simply zoned.

Having explored the behavior of these simulations under a range of mineral surface volumes that the fluid is capable of interacting with, we now fix this value to a running average of the previous 2.4 hours and proceed in exploring a range of surface areas. This bounds the original baseline conditions of the first set of simulations, and also influences the timeseries of the bulk fluid phase concentrations (Fig. 2A). As expected, a higher surface area of $10.0 \mathrm{~cm}^{2} / \mathrm{g}$ allows the system to establish equilibrium faster, whereas the lower $0.1 \mathrm{~cm}^{2} / \mathrm{g}$ value prolongs the departure from equilibrium. The corresponding isotope ratios of the fluid and the newly formed solid surface are strongly influenced by this surface area dependence (Fig. 2B). Relative to the baseline value in which the fluid can 'see' all of the solid phase, the original simulation using a running average of 2.4 hours and a surface area of $1.0 \mathrm{~cm}^{2} / \mathrm{g}$ is still illustrated with the same dashed blue line as in Fig. 1B.
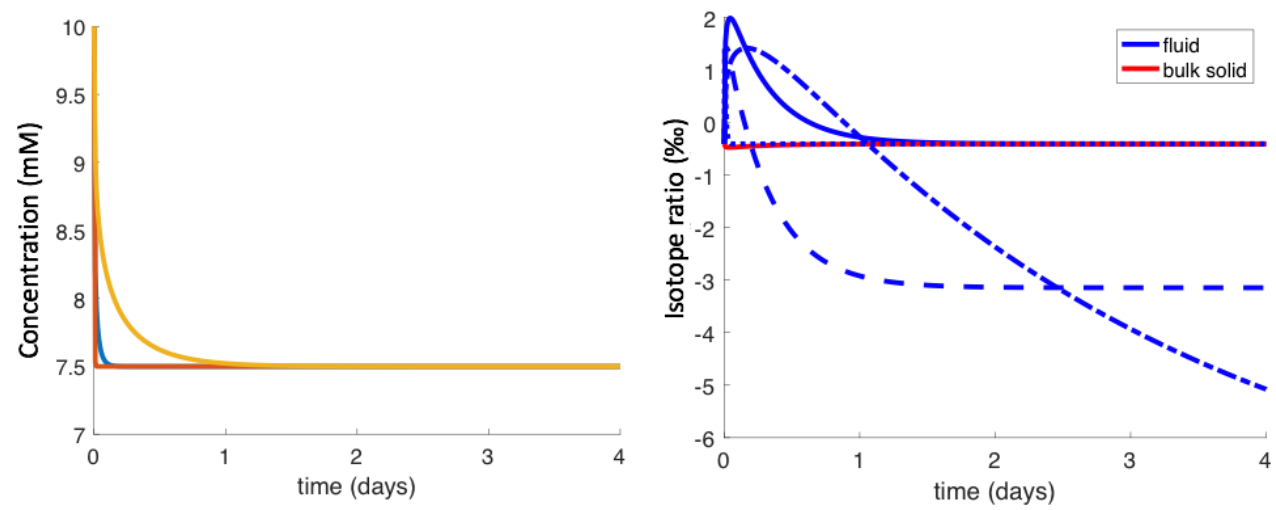

Fig. 2. Timeseries of simulated (A) fluid phase concentration of the initially oversaturated system for multiple mineral surface areas and (B) the corresponding isotope ratios of the fluid phase (blue) and the bulk solid phase (red). Multiple fluid phase simulations are shown for the isotope ratios which correspond to exchange with the bulk mineral (solid line), and exchange with solid phase precipitated over the prior 2.4 hours using a mineral surface area of $1.0 \mathrm{~cm}^{2} / \mathrm{g}$ (dashed line), $10.0 \mathrm{~cm}^{2} / \mathrm{g}$ (dotted line) and $0.1 \mathrm{~cm}^{2} / \mathrm{g}(\mathrm{dot}$ - dash line).

In addition to the prior simulation we now include the high surface area simulation, in which the period of time where the fluid is fractionated kinetically and becomes enriched is extremely short. As a result, by the time 2.4 hours has been established the isotope ratio of the fluid has already reequilibrated with the bulk solid, and thus behaves as if the entire mineral is available for isotopic exchange. This result is consistent with the inference that high surface area systems expose more of the overall mineral volume to water-rock interaction and thus lead to a homogeneous solid phase that reflects an isotopically equilibrated system. As a point of comparison, we also include a low surface area case, in which the overall period of time where the system is out of equilibrium is extended to approximately one day. As a result, the running average isotopic composition of the newly formed solid strongly influences the fluid, resulting in values that appear very far from equilibrium.

An important additional feature of this result is that if this system were open, such that fluid was continually advecting past this mineral surface, the extent to which the distinct 
solid surface isotope ratio would isolate the interior mineral from isotopic exchange following formation should be surface area dependent. Furthermore, a strong influence is anticipated as a result of the relative water/rock ratio, as the sparingly low mass of solute compared to the solid phase should result in a stronger influence on fluid phase compositions as the volume of fluid is decreased. In total these results suggest that the geometry of solid phases plays an important role in the preservation of isotope ratios and the extent to which they (1) record and (2) retain the isotopic signatures of water-rock interaction.

\section{References}

1. S. Brantley, J.D. Kubicki, A.F. White, Kinetics of Water Rock Interaction (Springer, 2008)

2. D. Porcelli, M. Baskaran, Handbook of Environmental Isotope Geochemistry, (Springer, 2011).

3. T.E. Cerling, Earth Planet. Sci. Let., 71 (1984).

4. B.L. Beard, C.M. Johnson, J.L. Skulan, K.H. Nealson, L. Cox, H. Sun. Chem. Geol., 195 (2003).

5. J.G. Wiederhold, S.M. Kraemer, N. Teutsch, P.M. Borer, A.N. Halliday, R. Kretzschmar. Environ. Sci. Technol, 40 (2006).

6. N.E. Jemison, A.E. Shiel, T.M. Johnson, C.C. Lundstrom, P.E. Long, K.H. Williams. Environ. Sci. Technol., 52 (2018).

7. D.J. DePaolo, Geochim. Cosmochim. Acta, 75 (2011)

8. M.S. Fantle, Geochim. Cosmochim. Acta, 148 (2015)

9. V. Mavromatis, Q. Gautier, O. Bosc, J. Schott, Geochim. Cosmochim. Acta, 114 (2013)

10. V. Mavromatis, I.A. Bundeleva, L.S. Shirokova, C. Millo, O.S. Pokrovksy, P. Bénézeth, M. Ader, E.H. Oelkers, Chem. Geol. 404 (2015)

11. V. Mavromatis, K. van Zuilen, B. Purgstaller, A. Baldermann, T.G. Nägler, M. Dietzel. Geochim. Cosmochim. Acta, 190 (2016)

12. V. Mavromatis, A.L. Harrison, A. Eisenhauer, M. Dietzel, Geochim. Cosmochim. Acta, 218 (2017)

13. C.R. Pearce, G.D. Saldi, J. Schott, E.H. Oelkers. Geochim. Cosmochim. Acta, 92 (2012)

14. E.H. Oelkers, U.N. Berninger, A. Pérez-Fernàndez, J. Chmeleff, V. Mavromatis, Geochim. Cosmochim. Acta, 226 (2018)

15. J.L. Druhan, C.I. Steefel, K.H. Williams, D.J. DePaolo, Geochim. Cosmochim. Acta, 119 (2013)

16. N. Fernandez, J.L. Druhan, Geochim. Cosmochim. Acta, (to be published)

17. J.L. Druhan, C.I. Steefel, M.E. Conrad, D.J. DePaolo, Geochim. Cosmochim. Acta, 124 (2014)

18. C.I. Steefel, J.L. Druhan, K. Maher, Proc. Earth Planet. Sci, 10 (2014)

19. J.L. Druhan, S.T. Brown, C. Huber, Reviews In Mineralogy and Geochemistry (2015) 